\title{
Khan Academy: Fortalecimiento del aprendizaje de Cálculo I en estudiantes universitarios.
}

\section{Khan Academy: Strengthening Calculus I Learning in College Students.}

\author{
Angel Salvatierra Melgar \\ Universidad Privada del Norte, Lima. Perú \\ ORCID: https://orcid.org/0000-0003-2817-630X \\ Sonia Romero \\ Universidad Femenina del Sagrado Corazón, Lima. Perú \\ ORCID. https://orcid.org/0000-0002-9403-410X \\ Linda Shardin Flores \\ Universidad Privada San Juan Bautista, Lima, Perú \\ ORCID: https://orcid.org/0000-0002-1279-8975
}

*Correspondence

Email: angel.salvatierra@upn.pe
Cite as:

Salvatierra, A., Romero, S., \& Shardin, L. (2021). Khan Academy: Fortalecimiento del aprendizaje de Cálculo I en estudiantes universitarios. Propósitos y Representaciones, 9(1), e1042. Doi: http://dx.doi.org/10.20511/pyr2021.v9n1.1042 


\section{Resumen}

El estudio puesto a consideración, muestra la importancia del uso del Khan Academy (KA) en el aprendizaje de Cálculo I en estudiantes universitarios de las Escuelas Profesionales de Ingeniería. Por las peculiaridades, la investigación abraza el enfoque cuantitativo, con diseño experimental de tipo cuasiexperimental con pretest y postest. La muestra fue de tipo no probabilístico intencional, desprendido en el grupo experimental constituidos por 93 estudiantes; mientras que, el grupo de control por 82 estudiantes de las carreras de ingeniería. Con respecto al instrumento, se asumió las pruebas pedagógicas validadas por 5 jueces representados por docentes del área de Cálculo I. Después de la experimentación que tuvo lugar en 10 sesiones de aprendizaje, se arribó a la conclusión. El uso de la plataforma del Khan Academy permitió promover el aprendizaje del Cálculo I en los tópicos de la derivada, los estudiantes que conformaron el grupo experimental, obtuvieron logros académicos considerables, además demostraron mayor confianza, autonomía y motivación durante el aprendizaje. Desde el aspecto tecnológico, el uso de la plataforma en línea, permitió rastrear las interacciones del estudiante al manipular las herramientas e interactuar con las actividades de la plataforma

Palabras clave: Khan Academy; Calculo Diferencial; Habilidades matemáticas; Aprendizaje virtual

\section{Summary}

Higher Education in Cuba is a particular transformational process; indeed, the development of knowledge in the society requires new demands in the universities. One of this demand is precisely the educational inclusion of young university students, for the achievement of the necessary professional skills in their performances. Particularly, those who have a diagnosis on stuttering and the ones who are studying pedagogical careers, need special attention by a language specialist (speech therapist) so that they can achieve their independence required by the exercise of the profession from a quality education. The speech therapy cabinet office is an excellent place to favor the educational inclusion of young university students with a diagnosis of stuttering, however, there is a limited level of knowledge by teachers, students and managers about the role they play in this regard. This article is aimed at evaluating the work that is done from the speech therapy cabinet of the Central University "Marta Abreu" de Las Villas (Villa Clara province, Cuba), to favor the educational inclusion of a young woman with Stuttering diagnosis. In order to achieve this purpose, an exploratory research was implemented through a case study, which constituted the initial phase of a further study. As a result, the diagnosis of the real state of the work that is carried out from the speech therapy cabinet was obtained, in relation to the educational inclusion of a young woman with a diagnosis of stuttering.

Keywords: Khan Academy; Diferential calculus; Mathematical skills; Virtual learning

\section{Introducción}

El cálculo diferencial es una asignatura dispuesta en las carreras de Ingeniería, Ciencias y ramas afines, ya que su aplicación responde a las necesidades profesionales con el fin de minimizar y maximizar procesos dentro de la producción, en aspectos logísticos, en optimizaciones del uso de materiales y en aplicaciones físicas. Además, involucra el estudio de funciones, las interpretaciones de la recta secante y pendiente a lo largo de una figura y las formas de representar las variables relacionadas, la razón de cambio y las aproximaciones, quienes juegan un papel importante en la formación profesional para la comprensión de los fenómenos relacionado a los conceptos de la diferencial aplicadas a sus campos profesionales. 
Dentro del ámbito del aprendizaje de la matemática, los estudiantes de nivel superior universitario experimentan diversos problemas de índole emocionales, social, económico, etc. Al respecto, Delgado et al. (2017) manifestaron que la ansiedad es uno de los problemas más comunes de los estudiantes. Asimismo, la procedencia de la Institución Educativa y la modalidad, la cantidad de veces que ha llevado el curso, las habilidades lógicas matemáticas, las competencias propias de la asignatura que se encuentran asociadas a diversos estilos de aprendizaje del estudiante, entre otros aspectos repercuten en el rendimiento académico del nivel universitario. Por su parte, Hoffman et al. (2017), consideraron que el rendimiento académico varía significativamente según las características académicas y sociodemográficas de los estudiantes; sin embargo, a medida de las interacciones en los nuevos círculos de aprendizaje, ellos logran adaptarse y asumir los retos impuesto para las diferentes asignatura como parte de su formación profesional.

El aprendizaje de las derivadas, es secuencia de los conceptos de límites de función, en este tópico, los estudiantes adquieren las capacidades y la habilidad para los cálculos de las derivadas y así aplicar los conceptos y propiedades en la solución de problemas orientados a su formación profesional. Sin embargo, no todos logran esta habilidad, debido a problemas de formación básica y de conectar los conocimientos previos con el nuevo aprendizaje. La formación básica de los conceptos matemáticos es evidente en los resultados de los exámenes que frecuentemente emite el Estado y otras entidades. Estos resultados, obstaculizan el aprendizaje del cálculo diferencial al registrarse carencias de las nociones de habilidades lógicas matemáticas. Además, a esta problemática se suma las metodologías y estrategias didácticas de los docentes, las cuales pueden convertirse en experiencias positivas o negativas para los estudiantes.

El abordaje simplista al Cálculo, obstaculiza la comprensión de conceptos matemáticos, que repercuten en la resolución de problemas referidos al cálculo. Incluso, los conceptos simplistas y superficiales conllevan al estudiante a la confusión durante la solución de ejercicios y problemas. (Valencia y Valenzuela, 2017). Para la resolución de ejercicios y problemas, al inicio es recomendable la contextualización para su comprensión y para abordar problemas variados, en estos escenarios se distinguen cuestionamientos como: problemas matemáticos convencionales; que procuran poner en juego los conocimientos previos de índole heurístico, que direccionan hipótesis y establecen modelos que explican matemáticamente cómo se comporta un fenómeno. Los modelajes matemáticos se fundamentan sobre los conocimientos matemáticos que permiten su aplicabilidad a casos concretos, estimulando la algoritmización y la aplicabilidad práctica en casos similares.

Según Portilla (2018) los modelos permiten minimizar procedimientos y obtener resultados concretos, fidedignos y lógicos. Desde esta perspectiva, el estudio se fundamenta en el aprendizaje teórico del Calculo I como parte de la formación profesional del futuro ingeniero, con aplicaciones en tres vertientes: a) la argumentación teórica de los temas de la derivada y las integrales indefinidas y, la resolución de ejercicios, b) las aplicaciones de las derivadas a casos de su carrera profesional, y c) el estudio de caso con aplicación práctica basado a un modelo.

El cálculo diferencial, se caracteriza principalmente por presentar un componente visual e intuitivo aplicado fundamentalmente en la física y en geometría. Con relación a ello, Vrancken y Engler (2014) refirieron que el análisis y estudio de la variación de casos dinámicos condujo a visualizaciones e intuitivos de los fenómenos, permitiendo la comprensión de la variación en tres aspectos básicos: Razón media de cambio, el cambio y razón instantánea de cambio, que hizo 
posible describir, cuantificar y pronosticar la rapidez de los fenómenos de la naturaleza a situaciones cotidianas.

Para Parra et al. (2019)La aplicación de la razón de cambio instantánea , permite la adquisición de las nociones del crecimiento poblacional con restricciones dentro del cálculo diferencial. De la misma forma, Dominguez et al. (2019) sostuvieron que para la comprensión del concepto de la derivada en ingenierías y ciencias es indispensable los conceptos previos de: números reales, función y limites; así como, sus representaciones y relaciones gráficas. Estos tópicos orientan a la comprensión de los conceptos de cinemática, el análisis de los gráficos de una función, velocidad (primera derivada) y aceleración (segunda derivada), las aproximaciones y las razones de cambio. Sin embargo, Morales et al. (2018) proponen cuatro pasos para el aprendizaje de la derivada: El propósito, saberes previos respecto a la derivada, el aprendizaje de la derivada por las definiciones de los límites y el comportamiento de la pendiente de una función en un punto dado.

La existencia de la derivada de una función $\mathrm{f}$, en un número real " $\mathrm{a}$ ", es definido como: $f^{\prime}(x)=\lim _{x \rightarrow a} \frac{f(x)-f(a)}{x-a}$, que brinda información de variación $\mathrm{f}$ en entorno de "a", referido a la variación de $f(x)-(a)$ (Parra, Gordillo y Pinzón, 2019). Estos argumentos preliminares, permitieron el desarrollo teórico-práctico de la aplicación a diversos casos de cambio dentro del contexto de la física, la variación de velocidad, problemas de movimiento y tiempo de alcance, problemas de fluidos en conos invertidos, el comportamiento de la aceleración de una partícula, representación gráfica de funciones quien permitió la visualización de la razón de cambio media, la pendiente de recta y aproximaciones.

El aprendizaje de las derivadas en estudiantes universitarios es un foco de atención por sus múltiples aplicaciones; dado que, muestra interés en diversos campos profesionales de las Ciencias e Ingeniería. Por lo tanto, para la sistematización del concepto, es necesario la modelación matemática y la aplicación de métodos heurísticos que permiten la comprensión, análisis de problemas de razones y diferencias entre magnitudes infinitamente pequeñas (Rizo y Campistrous, 1999); entre estos métodos, se cuenta el uso de representaciones gráficas, datos numéricos o el uso de diagramas con el fin de llegar a estructuras más simples para su interpretación. Caseres et al. (2019) refieren que el concepto de infinitesimal representa la diferencia entre dos valores de una misma variable con longitudes tan pequeñas tendiendo al cero, aplicadas a fenómenos y situaciones que permiten minimizar o maximizar los diversos sucesos.

Por la gran utilidad del Cálculo Diferencial, las universidades ofrecen cursos de extensión, seminarios o círculos de estudio guiados por sus docentes, con la finalidad de responder a los desafíos profesionales, implementando resoluciones de problemas, asesorías, tutorías, talleres de refuerzo, clases magistrales, implementación metodológica activa como el ABP acompañados de trabajos prácticos, implementando al uso de nuevas tecnologías en el proceso de enseñanza-aprendizaje. Todo este evento con la finalidad de despertar el interés, la motivación y descender el índice de repitencia o descensión que es muy frecuente en las aulas universitarias. Por tanto, con el fin de reforzar el aprendizaje, se ha puesto a consideración el uso de la plataforma que se muestran a continuación. 


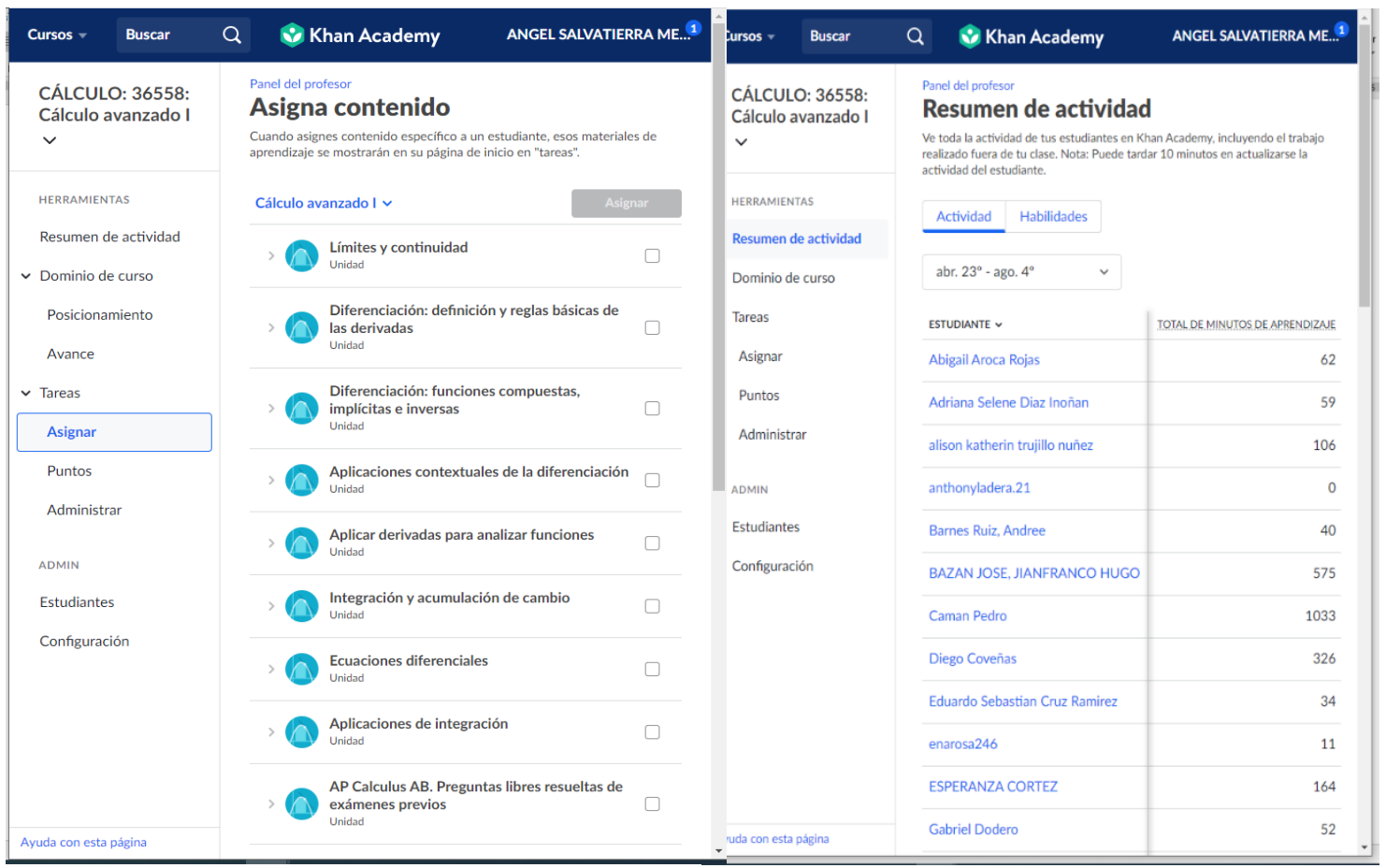

Figura 1. Captura del interfaz de contenidos asignados y el resumen de actividades del tópico de Calculo I.

En la captura de pantalla, se aprecian los títulos de los contenidos del tópico del Calculo I asignados a estudiantes durante la estancia de la experimentación, además se visualiza un fragmento de la asistencia de estudiantes perteneciente al aula con código de 36558, como también los minutos de interacción con el Khan Academy (KA) para cada tópico de la asignatura.

\begin{tabular}{|c|c|c|c|c|c|c|c|}
\hline \multirow{6}{*}{$\begin{array}{l}\text { Cursos - Buscar } \\
\text { CÁLCULO: 36558: } \\
\text { Cálculo avanzado I } \\
\end{array}$} & \multirow{2}{*}{ Q } & \multirow{2}{*}{8 Khan Academy } & Intentado ( $(6)$ & \multicolumn{2}{|l|}{ Familiar (4) } & \multirow{2}{*}{$\begin{array}{l}\text { Competente (16) } \\
\text { Barnes Ruiz, Andree }\end{array}$} & \multirow{2}{*}{ Dominado } \\
\hline & & & Gabriel Dadero & Abigail Aroca & & & \\
\hline & \multirow{5}{*}{\multicolumn{2}{|c|}{$\begin{array}{l}\text { Panel del profesor } \\
\text { Resumen de actividad } \\
\text { Ve toda la actividad de tus estudiantes en Khan Academy, incluyendo el trabajo realizado fuera de } \\
\text { tu clase. Nota: Puede tardar } 10 \text { minutos en actualizarse la actividad del estuciante. }\end{array}$}} & Leonardo Vega & \multirow{4}{*}{\multicolumn{2}{|c|}{$\begin{array}{l}\text { Adriana Selene Diaz .... } \\
\text { Jaimes Rojas Cesar A... } \\
\text { Jeremyt Modragón C... }\end{array}$}} & \multirow{2}{*}{\multicolumn{2}{|c|}{ BAZAN JOSE, JIANF... }} \\
\hline & & & Lucia Milagros Uchar... & & & & \\
\hline & & & Sharon Bermudez & & & - Eduardo Sebastian C... & \\
\hline & & & Sofia Galindo Ruiz & & & enarosa246 & \\
\hline \multirow{2}{*}{ HERRAMIENTAS } & & & Zambrano Ramos Ju... & & & ESPERANZA CORTEZ & \\
\hline & \multicolumn{2}{|c|}{\begin{tabular}{l|l} 
Actividad & Habilidades \\
\end{tabular}} & & & & JOSELI DAYANNA & \\
\hline \multirow{2}{*}{ Resumen de actividad } & \multirow{3}{*}{\multicolumn{2}{|c|}{ Cálculo avanzado I }} & & & & kelly Barreta Mamani & \\
\hline & & & & & & Llantoy Castillo Cesa... & \\
\hline - Dominio de curso & & & & & \multirow{2}{*}{\multicolumn{3}{|c|}{ Moreno Bazán Brisa }} \\
\hline Posicionamiento & \multicolumn{2}{|c|}{ Cálculo avanzado I } & & & & & \\
\hline Avance & \multicolumn{2}{|c|}{10 unidades 164 habilidades } & \multicolumn{5}{|c|}{ - Derivadas de funciones inversas } \\
\hline - Tareas & \multicolumn{2}{|c|}{ > Límites y continuidad (34 habilidades) } & \multirow{2}{*}{\multicolumn{5}{|c|}{$\begin{array}{l}\text { - Derivadas de funciones trigonométricas } \\
\text { inversas }\end{array}$}} \\
\hline Asignar & \multirow{2}{*}{\multicolumn{2}{|c|}{ > Diferenciación: definición y reglas básicas de las derivadas (23 habilidades) }} & & \multicolumn{4}{|c|}{ inversas } \\
\hline Puntos & & & \multirow{2}{*}{\multicolumn{2}{|c|}{$\begin{array}{l}\text { - La derivada de funciones: encontrar el } \\
\text { errar }\end{array}$}} & & & \\
\hline \multirow[t]{2}{*}{ Administrar } & \multicolumn{2}{|c|}{ > Diferenciación: funciones compuestas, implícitas e inversas (15 habilidades) } & & & & & \\
\hline & \multicolumn{2}{|c|}{ > Aplicaciones contextuales de la diferenciación (14 habilidades) } & \multicolumn{2}{|c|}{ - Manipular funciones antes de derivarlas } & & & \\
\hline Estudiantes & $>$ Aplic & derivadas para analizar funciones (18 habilidades) & \multicolumn{2}{|c|}{ Derivar con varias reglas: estrategia } & & | | | & \\
\hline Configuración & > Integ & ión y acumulación de cambio (30 habilidades) & - Diferenciar con el uso de $\mathrm{v}$ & rarias reglas & & 1 & \\
\hline & > Ecuac & nes diferenciales (11 habilidades) & - Segundas derivadas & & 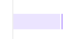 & bu & \\
\hline & > Aplic & ones de integración (19 habilidades) & $\begin{array}{l}\text { - Segundas derivadas (ecuac } \\
\text { implíitas) }\end{array}$ & ciones & - & & \\
\hline & 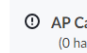 & $\begin{array}{l}\text { ulus AB. Preguntas libres resueltas de exámenes previos } \\
\text { dades) }\end{array}$ & - Derivadas con disfraz & & b & & \\
\hline
\end{tabular}

Figura 2. Captura de pantalla, resumen de las habilidades y de los niveles de logros de los estudiantes del tópico de Calculo I. 
En la interfaz, se aprecian los niveles de las habilidades obtenidas durante las interacciones del estudiante en los tópicos del Calculo I reportado por la plataforma. Estos niveles, son clasificados como: intentados y dominados, en donde se diferencian los logros por colores y por tópicos. Además, se observan el número de habilidades desarrolladas por los tópicos como resultados de la participación de los estudiantes, que se sitúan en los tres niveles: Intentado, Familiar y Competente. En la interfaz también se aprecia que, buen grupo de estudiantes muestran un nivel competente en diferentes tópicos. En el tópico de: Diferenciar con el uso de varias reglas, los estudiantes obtuvieron mayor participación de interacción con el KA y también mayor dominio al tema arribando al nivel competente y familiar. De igual manera, al respecto del tópico de: Derivar con varias reglas estrategias, se tiene mayor nivel de logro, es decir, nivel competente. Estos resultados, indican que los estudiantes presentan interacción con los tópicos mediante la visualización de videos, práctica con los ejercicios y problemas resueltos, la asertividad resolutiva de los ejercicios y problemas propuestos.

\section{Aprendizaje virtual}

Debido al aislamiento que se vive a nivel mundial, la práctica de las acciones educativas de la enseñanza- aprendizaje ha migrado a la virtualidad cobrando importancia e interés por el uso de las herramientas de las Tecnologías de la Información y la Comunicación (TIC). Hoy más que nunca, ha tomado importancia y utilidad en diferentes sectores, más aún dentro de contextos educativos que viene contribuyendo a la inclusión y acceso de información abierta gracias al uso de las herramientas de las TIC (Diaz y Rueda, 2020). Con esto queda claro que, las TIC mejoran visiblemente la adquisición del conocimiento, que es transmitido en los resultados de las habilidades matemáticas especialmente con el uso de la herramienta web KA, es allí, donde los estudiantes interactúan en diversas circunstancias, tanto para: reforzar sus aprendizajes, practicar ejercicios y problemas con los seguimientos, someterse a una auto evaluación y comprobar el auto dominio de un determinado tópico.

La época que nos está tocando vivir tiene una característica principal para el estudiante en comparación en épocas pasadas. Sobre esto, Álvarez (2018) manifestó que estos movimientos culturistas son propios de esta generación del milenio que responden a los procesos de la globalización y los cambios culturales producto de las interacciones de los medios de comunicación de manera acelerada asociada a la tecnología. El uso de las TIC permite lograr los estándares de calidad y por ende, permite a su vez, el desarrollo tecnológico e innovación para el bienestar y progreso del estudiante en diferentes niveles conducentes a una alfabetización digital generando así la mejora continua en el ámbito de la enseñanza-aprendizaje; es decir, estos cambios acelerados contribuyen a una nueva forma de trabajo en los escenarios educativos, una forma diferente de enseñar y aprender.

La aparición de herramientas y plataformas de libre acceso en el campo educativo, está permitiendo potencializar el uso y adquirir nuevas formas de aprender, tal es el caso del aprendizaje ubicuo (María et al., 2015). De esta manera, son los universitarios quienes gestionan sus aprendizajes de manera libre y espontánea desde donde se encuentran físicamente ubicados a través del uso de dispositivos móviles. Esta brecha digital formativa para el autoaprendizaje en estos escenarios, ha tomado un espacio inmenso y está ganando terreno por la gran cantidad de información abierta; en el caso específico, en los tópicos del Calculo I. En palabras de Fonseca y Alfaro (2018) el cálculo diferencial e integral es parte de los planes de estudios de las carreras vinculadas a las ciencias exactas y naturales Para Lois y Milevicich (2008) la algebrización del cálculo diferencial e integral se pone de manifiesto con el enfoque algebraico y reduccionista de 
la enseñanza que contempla las operaciones algebraicas con límites, derivadas e integrales; en éste sentido, los estudiantes logran comprender el concepto de las derivadas a partir de los conceptos previos tratados sin dificultad para el aprendizaje.

Los cambios frecuentes de la práctica docente y el aprendizaje del estudiante a raíz de la inserción de las TIC, contribuyen al uso y aplicación de la información existente, mostrándose una nueva forma de aprendizaje. Rueda y Guzmán (2018) indican que el uso de la tecnología de información se ha convertido en una herramienta poderosa en el proceso de aprendizaje en diferentes áreas y es parte indispensable de nuestra vida académica y cultural, su existencia ha generado expectativas por el tipo de contenido variado en función al contexto y nivel de complejidad de los temas de interés.

El estudio, toma como referencia a los aportes pedagógicos del aprendizaje ubicuo de (Siemens, 2005) debido al aislamiento social ocasionado por la pandemia Covid-19. En condiciones normales, el desarrollo de la asignatura tiene carácter presencial; sin embargo, por el motivo explicado, se viene desarrollando de manera virtual y es fortalecido con materiales expuestos en herramientas y plataformas con libre acceso como videos, libros electrónicos, herramientas, calculadoras, simuladores, etc. Para el estudio, se ha asumido al uso del KA por las características que permiten afianzar el aprendizaje de manera oportuna en esta sociedad del conocimiento, y al uso de la tecnología que permite el progreso personal y social, conducentes a la alfabetización digital y los logros de competencias digitales para la administración y procesamientos de la información.

Según Rodríguez et al. (2014) el Khan Academy es una plataforma de libre acceso para el apoyo del aprendizaje interactivo de personas interesadas en temáticas de ciencias. Ofrece más de 10000 videos educativos en línea. Últimamente incrementaron temas de estadística donde cada tópico incluye ejercicios y problemas con solución en tiempo real, con ejercicios con solucionario para que el interesado compruebe sus logros. Es importante mencionar también que la plataforma presenta cuatro componentes principales como: datos, videos, ejercicios y una comunidad de usuarios que permite interactuar y dejar sus comentarios al proceso de solución.

Por la gran interactividad y estructura del KA, esta permite la motivación del estudiante a secuencializar un tema tras otro, invitando a generar hábitos de estudio, previas recompensas con medallas, meteorito, luna, tierra, es decir, íconos virtuales cada vez que logran habilidades desafiantes de aprendizaje, en otras palabras, las condecoraciones que otorga la plataforma son por las recompensas al desafío. Otra fortaleza de la plataforma, es la generación estadística personalizada para el docente, que permite el seguimiento a los estudiantes en cuanto a los tiempos y a la inserción a la herramienta del KA.

Como expresa Yassine et al. (2020), el KA es un recurso educativo abierto y ampliamente utilizado por estudiantes y profesores en diferentes temas del aprendizaje. A medida que el estudiante interactúa con la plataforma y logra las habilidades adecuadas, la plataforma los clasifica por niveles: Nivel 1, indica que previamente practicó y horas después fue reforzada; nivel 2 estuvo en nivel 1 y después practicó mediante reto de dominio; y, el dominado, se refiere al conjunto de habilidades en el nivel 2 y horas, días y meses después fueron reforzadas de forma continua. Este sistema métrico permitió al estudiante afianzar sus capacidades en la resolución de los ejercicios de manera individual y retroalimentada continuamente que brinda la plataforma. Finalmente, Yassine et al. (2020) agrega que el rápido crecimiento y uso de las redes de 
aprendizaje, como la plataforma del KA, es ampliamente visualizado por su peculiaridad ante los ejercicios y problemas que permitió el aprendizaje oportuno en línea con acceso abierto.

\section{Método}

Por las características descritas del estudio, la investigación se encuentra dentro del enfoque cuantitativo, de diseño experimental de tipo cuasiexperimental con pretest y postest (Hernández et al., 2014). La muestra asumida para el estudio, fue de tipo no probabilístico intencional, ya que los grupos intactos fueron conformados de acuerdo a su matrícula y al acceso voluntario a la interacción con la plataforma KA. Los integrantes del grupo experimental fueron constituidos por 93 estudiantes, de los cuales 55 eran varones y 38 eran mujeres; mientras que, el grupo de Control estuvo constituido por 82 estudiantes; 44 de ellos varones y 38 mujeres. La muestra estuvo conformada por 175 estudiantes considerados como incluidos y 25 estudiantes fueron excluidos por asistencia irregular a las sesiones de aprendizaje y que no lograron completar los ejercicios propuestos en el KA. Con respecto a los instrumentos de recogida de los datos, se sujetaron a las pruebas pedagógicas de la validez de contenido por 5 jueces representados por los docentes del área de Cálculo I. Los datos obtenidos fueron procesados con el software SPSS_V25, para luego comparar los resultados antes y después del GE y GC, mediante el estadístico de distribución normal $\mathrm{Z}$ al nivel de significación de $\alpha=0.05$ para muestras independientes.

Se desarrolló la experimentación con 5 aulas, cada una constituida por 40 estudiantes de diferentes escuelas profesionales de ingeniería: Civil, Sistemas Computacionales, Industrial, Agroindustrial, Ambiental, Minas, electrónica, Logística y transporte, Geológica, Mecatrónica y Empresariales; en el turno mañana y tarde; bajo el siguiente procedimiento: identificado el estudiante, se invitó a registrarse de forma voluntaria a la plataforma del KA, procediéndose a la evaluación de entrada con el fin de identificar las habilidades y los conocimientos previos de las temáticas de la asignatura.

En cuanto a la disposición a la plataforma, los estudiantes muestran acceso al Blackboard, donde se encuentran alojados los materiales de clase distribuidos por semana, como: clases teóricas, prácticas y hojas de ejercicios. Además, se muestran los enlaces con información complementaria por semana, con acceso a la plataforma del KA en relación a las clases tratadas. La participación dentro de este último se consideró desde el tablón de la misma, interactuaron con las prácticas, videos interactivos y la retroalimentación de 4 a 5 ejercicios disponibles. Las actividades interactuadas en la plataforma, se observan en el portal de reportes como: el tiempo de acceso, habilidades adquiridas, nivel alcanzado por diversos colores, porcentaje de acierto por cada tópico y ejercicios resueltos. Esta secuencia se repitió hasta el final del tópico de las derivadas. Sin embargo, los estudiantes del grupo de control, fueron aquellos que no se visualizaban en la plataforma del KA, posiblemente hicieron uso otros medios de información para el logro de sus aprendizajes.

\section{Resultados}

Desde los enunciados de los marcos conceptuales y metodológicos asumidos en el estudio, a continuación, se muestran los resultados obtenidos de la experimentación, que permitió la comparación y confrontación de los logros entre ambos grupos de estudio. 
Tabla 1

Estadísticos y parámetros de los logros académicos entre los grupos de estudio

\begin{tabular}{|c|c|c|c|c|c|c|c|c|}
\hline Tes & Grupo & & General & $\begin{array}{c}\text { Zc; } \\
\text { p_valor }\end{array}$ & Masculino & $\begin{array}{c}\text { Zc; } \\
\text { p_valor }\end{array}$ & Femenino & $\begin{array}{c}\text { Zc; } \\
\text { p_valor }\end{array}$ \\
\hline \multirow{2}{*}{ Pre test } & \multirow{2}{*}{ Contro (82) } & $\bar{x}$ & 11.51 & \multirow{4}{*}{$\begin{array}{c}Z=1.106 \\
p \_ \text {valor }= \\
0.328\end{array}$} & 11.16 & \multirow{4}{*}{$\begin{array}{c}Z=0.791 \\
p \_ \text {valor }= \\
0.530\end{array}$} & 11.29 & \multirow{4}{*}{$\begin{array}{c}Z=1.878 \\
p \_ \text {valor }= \\
0.350\end{array}$} \\
\hline & & $s$ & 0.64 & & 3.35 & & 2.032 & \\
\hline \multirow{3}{*}{ Pre test } & \multirow{3}{*}{$\begin{array}{c}\text { Experimental } \\
(93)\end{array}$} & $\bar{x}$ & 11.01 & & 11.56 & & 10.21 & \\
\hline & & $\mathrm{s}$ & 4.047 & & 3.326 & & 4.844 & \\
\hline & & sig & & N.S & & N.S & & N.S \\
\hline \multirow{2}{*}{ Pos test } & \multirow{2}{*}{ Contro (82) } & $\bar{x}$ & 15.13 & \multirow{4}{*}{$\begin{array}{c}Z=3.212 \\
p \_ \text {valor }= \\
0.00\end{array}$} & 15.41 & \multirow{4}{*}{$\begin{array}{c}Z=2.256 \\
p \_ \text {valor }= \\
0.01\end{array}$} & 14.83 & \\
\hline & & $s$ & 2.74 & & 2.67 & & 2.67 & n valor= \\
\hline \multirow{3}{*}{ Pos test } & \multirow{3}{*}{$\begin{array}{c}\text { Experimental } \\
\qquad(93)\end{array}$} & $\bar{x}$ & 16.4 & & 16.25 & & 16.03 & 0.010 \\
\hline & & $\mathrm{s}$ & 2.49 & & 2.254 & & 2.824 & \\
\hline & & sig & & A.S* & & $S$ & & $S$ \\
\hline${ }^{2}(1$ & 1.96 & $\alpha=$ & & mon & vo & III & . & ficativo \\
\hline
\end{tabular}

Los resultados que se muestran en la tabla, permiten identificar las estadísticas de la media y la desviación típica de los 82 estudiantes que conformaron en grupo de control ( $\bar{x}=11.51$ ys $=$ $0.64)$, frente a los 93 estudiantes del grupo experimental $(\bar{x}=11.01$ y $s=4.047)$. Estos resultados del pre test, permitieron mostrar la similitud entre las medias; sin embargo, la desviación de grupo experimental es mayor que del grupo de control. Estos datos permiten la contrastación mediante la prueba de Distribución Normal (DN), que representa 1.106 frente al valor critico de $Z_{\left(1-\frac{\alpha}{2}\right)}=1.96$ a un nivel de significación estadística de $\alpha=0.05$, $(1.96>1.106)$. Además, el p_valor > 0.05, permitió afirmar la ausencia de diferencia de medias entre los resultados del pre test en la asignatura del Calculo I en ambos grupos, estas comparaciones permitieron manifestar que los estudiantes de nivel superior, presentaban habilidades matemáticas para comprender y absolver situaciones de Cálculo I.

Estos mismos comportamientos se presentan en las comparaciones de medias entre los grupos en el pre test con respecto al género. Los estudiantes de sexo masculino del grupo de control presentan la $(\bar{x}=11.16$ y $s=3.35)$ frente a los del grupo experimental de la $(\bar{x}=$ 11.5 y $s=3.33$ ) apreciándose cierta similitud entre ambas estadísticas. La comparación por la prueba de la DN 1.96>0.79, además p_valor >0.05, asumimos la no existencia de diferencia entre los promedios por sexo masculino en el pre test. Este mismo comportamiento se aprecia en los resultados del sexo femenino; puesto que, el 1.96>1.88 con medias de 11.29 y 10.21 representando al grupo control y experimental, respectivamente y p_valor $>0.05$.

Identificados estos resultados, se desarrollaron las actividades tanto para el estudiante del grupo de control y experimental durante 10 semanas, en los tópicos de: Derivada, regla de cadena, ecuación de la recta normal y tangente, derivada de función inversa, regla de L’Höpital, derivadas implícitas, derivación paramétrica, tasa de cambio, funciones, criterio de la primera y segunda derivada, teorema de Rolle, optimización, linealidad y aproximación. Los estudiantes del grupo experimental hicieron uso de la plataforma del KA como refuerzo a los temas tratados; mientras que, los del grupo de control posiblemente hicieron uso de otros medios para su aprendizaje, los resultados se describen a continuación. 
Después del desarrollo de las sesiones de aprendizaje, los estadísticos obtenidos en el grupo de control son $(\bar{x}=15.13$ y $s=2.74)$ mientras en el grupo experimental $(\bar{x}=16.4$ ys $=$ 2.49) respectivamente. Por lo tanto, la comparación de estos, permite identificar el efecto de KA en 1.27 puntos de diferencia entre los promedios; además, las calificaciones del grupo experimental son más homogéneas que del grupo de control. Los resultados obtenidos permitieron la comparación de medias para muestra independientes de varianza homogénea, el valor critico de $Z_{\left(1-\frac{\alpha}{2}\right)}=1.96$ frente al $Z \mathrm{c}=2.61$, quienes permiten la comparación $1.96<3.21$, por su ubicación de la Zc dentro de la región critica derecha de la curva de distribución normal. Asimismo, se tienen al valor de significación estadística de p_valor de 0.01 frente al nivel de significación estadística de 0.05 ; p_valor $<0.05$. Es por eso que, estos resultados permiten afirmar, que el uso de la plataforma del (KA) promueve el aprendizaje de los temas del Calculo I.

Respecto a los resultados del post test por sexo. En cuanto al sexo masculino, se muestran los logros académicos en ambos grupos; sin embargo, la comparación de medias con la prueba de DN y al p_valor $0.01<0.05$, similar comportamiento se muestra en los resultados con respecto a los del género femenino. Estos resultados, permiten afirmar que, el uso de la plataforma del KA promueve el aprendizaje de los temas del Calculo I en estudiantes por género de los grupos de control y experimental, el KA, es un recurso educativo abierto y sus herramientas presentan impacto durante el aprendizaje por la variedad de ejercicios resueltos y videos donde se muestran los procedimientos resolutivos de problemas y ejercicios.

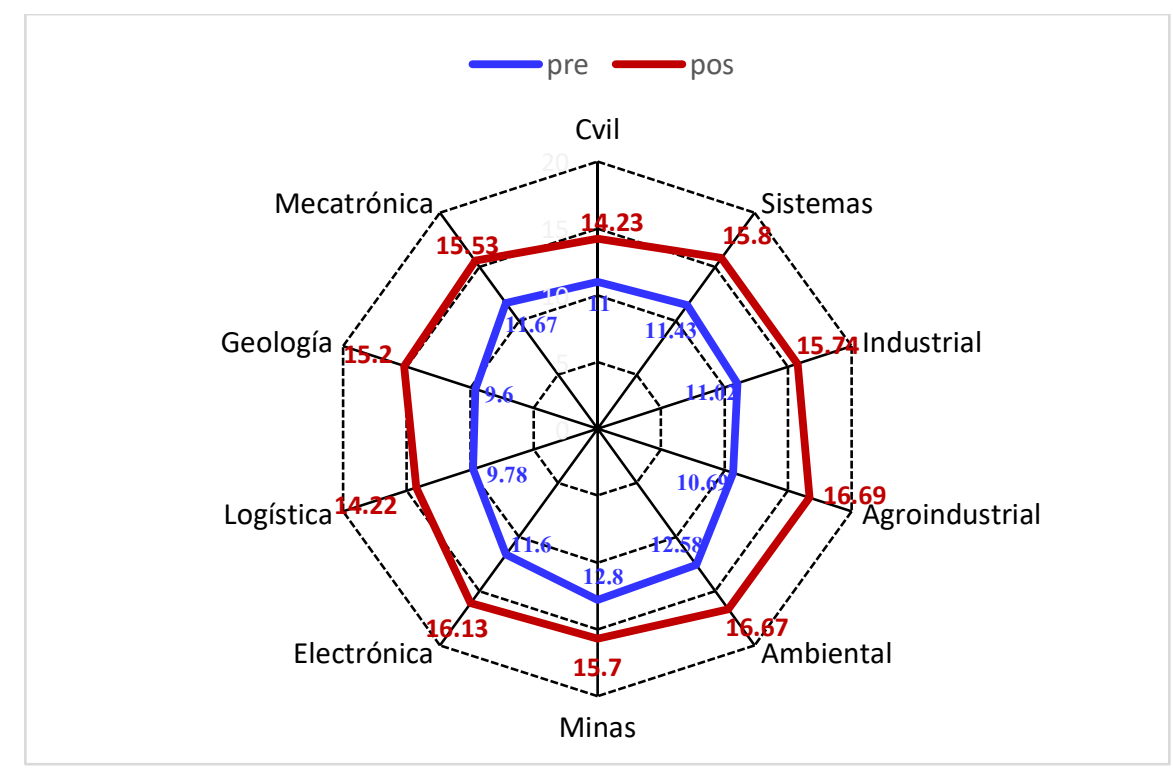

Figura 3. Diagrama de comparación de los promedios del pre y post test de los grupos de control y experimental

El diagrama radial muestra los logros obtenidos en el post test en comparación con el pre test con respecto a los promedios de las diferentes carreras profesionales. En lo que respecta a la carrera profesional con mayor logro académico mediante el uso del KA, es de Agroindustrial con un incremento de 6 puntos de promedios entre el post tes y pre tes, seguido por la carrera de Geología con 5.6 puntos. Asimismo, la carrera profesional de Industrial con 4.72 puntos, seguido por la carrera de Electrónica con 4.53 puntos. Sin embargo, los estudiantes de la carrera de Minas, presenta el menor promedio de 2.9 punto de comparación entre el post y pre test. En suma, existe una diferencia de promedios entre el post test y pre test de 4.374 puntos. 


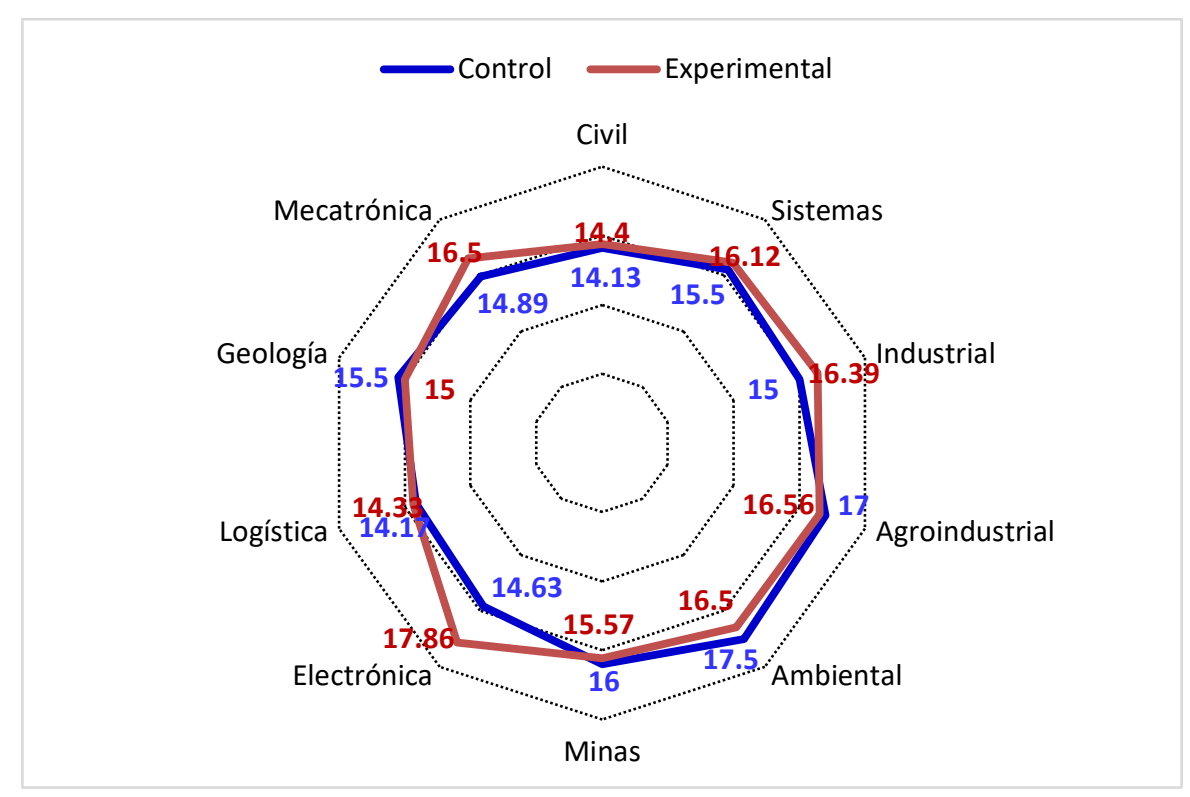

Figura 4. Diagrama de comparación de promedios del post test de los grupos de control y experimental

En el diagrama, se muestran los promedios del post test de las escuelas de ingeniería tanto del grupo de control y experimental. Las carreras profesionales de ingeniería en: Electrónica, Logística, Mecatrónica, Civil, Sistemas e Industriales, quienes obtuvieron logros académicos considerables en la asignatura de Cálculo I gracias al uso y refuerzos con la plataforma del KA. Los estudiantes de la carrera profesional de Electrónica, muestran mayor logro académico en comparación de promedios entre el grupo experimental y control con 3.23 puntos, posiblemente sea por la formación profesional que aprovecharon los recursos tecnológicos, ello muestra la importancia por los procedimientos resolutivos de manera familiar.

A consecuencia de la manifestación, los estudiantes de la carrera profesional de Mecatrónica presentan 1.611 puntos de comparación de promedios entre los grupos. De igual forma, los estudiantes de la escuela profesional de Industriales con 1.39 puntos de comparación de promedios entre ambos grupos de estudio, estos mismos comportamientos se observan en las carreras profesionales de ingeniería Civil, sistemas y Logística. Estos logros obtenidos, se deben al uso de la plataforma del KA en el aprendizaje del Calculo I en los tópicos de diferenciales; en cambio, los estudiantes que se encuentran en el grupo de control muestran logros académicos haciendo uso de otros recursos o medios, son los de Ingeniería Ambiental con un punto de diferencia entre los promedios del post test del grupo de control y experimental. Por último, se tienen a los alumnos de Geología, Agro industrial y Minas, que posiblemente hicieron uso de otros recursos académicos y tecnológicos que permitieron obtener calificativos superiores a los del grupo experimental dentro de las respectivas carreras profesionales.

\section{Discusión}

Los resultados obtenidos durante el estudio en comparación entre carreras profesionales, entre género que es visible y en pre test frente al post test durante el desarrollo del trabajo de experimentación. A su vez, se ha podido detectar la diferencia significativa gracias el uso del KA en la mayoría de las carreras profesionales; a pesar de los estudiantes de algunas carreras profesionales al no usarlo, en el KA obtuvieron calificaciones alentadores, debido a que los estudiantes universitarios adquieren estrategias variadas para el empoderamiento de la 
información, además presentan habilidades matemáticas para comprender y absolver situaciones de Cálculo I. (Mercedes y Hernández (2016) enfatizan que los estudiantes del nivel superior presentan competencias matemáticas y habilidades lógicas, quienes demuestran inteligencia fluida, hábitos de estudio y las actitudes hacia las Matemáticas.

En el estudio de Barahona(2014) se consideró un modelo de Regresión Múltiple, encontrando evidencia que las variables: género, estudio y trabajo, conformidad con la carrera, entre otros, presentan incidencia en los rendimiento académico del estudiante. Sin embargo, en el estudio, no se ha podido detectar diferencia significativa entre los promedios obtenidos por género, implicando el estudiante de ambos géneros presentan habilidades lógicas para el empoderamiento de los contenidos de Calculo. Ahora bien, las calificaciones del grupo experimental masculino son más homogéneas que del grupo de control. Según Muhammad et al. (2020) el uso de las plataformas de educación en línea, permiten rastrear los comportamiento académicos gradual de aprendizaje, asistido de manera libre y voluntaria. En el estudio de Vidergor y Ben-Amram, (2020) los estudiantes universitarios adquieren independencia y compromiso con su aprendizaje, ello explica la no existencia significativa entre la comparación de género.

En el estudio ha podido demostrar que el KA es una plataforma que permite afianzar y consolidar el aprendizaje de Calculo I en los temas puesto a experimentación. Por su parte Yassine et al. (2020), opinan que es un recurso educativo abierto donde sus herramientas presentan impacto durante el aprendizaje por la variedad de ejercicios resueltos y videos que muestran los procedimientos resolutivos de problemas y ejercicios, tal como se demostró en la carrera profesional de Agroindustrial con un incremento de 6 punto entre el promedio de post test y pre test. Por su cuenta, Chávez (2018) afirma que el uso de estrategias de aprendizaje en la obtención de notas finales en la asignatura de matemática son influenciadas y mejoradas por el uso de las plataformas o simuladores, en el caso específico del estudio con el KA.

En los hallazgos de Lou y Jaeggi (2020) la implementación tecnológica del KA para el aprendizaje muestra beneficios en comparación con el grupos de control. Además, permite la activación del conocimiento previo, que constituye un potencial tecnológico; sin embargo, Rossi et al. (2020) desarrolló una experiencia con docentes de Ingeniería centrado en el desarrollo de competencias académicas con el uso de software y plataformas, sus resultados permitieron evidenciar que el uso del KA u otros medios permiten buenos logros académicos.

En los diagramas radiales se muestra las comparación entre los promedios del post test de las escuelas de ingeniería, detectándose una comparación significativa en algunas escuelas profesionales. Mondal et al. (2019) examinó los procedimientos algorítmicos durante la solución de los problemas de la plataforma del KA y detectó alta correlación entre lo explicado en clase y las acciones desprendidas en los videos de la plataforma por su familiarización para quien desea aprender sobre los temas tratados en clase.

Los logros obtenidos con el uso de la plataforma del Khan-Academy, permitieron evidenciar el aprendizaje del Calculo I, en los aportes de (Jeong et al., 2019), identifica y analiza a 5538 entre videos y preguntas, distribuidos en diversos tipos de preguntas, nivel medio y alto; sin embargo, los estudiantes que se encuentran en el grupo de control, muestran logros académicos haciendo uso de otros recursos. Como lo hace notar Grisales (2018) el uso de recursos tecnológicos en clases de matemáticas tiene un impacto adecuado y urge la necesidad de articular los recursos de la TIC en los currículos de formación del profesional. Por su parte, Mercedes \& Hernández (2016) recomiendan el uso de otras plataformas similares a lo experimentado, ya que 
formaliza y consolida el aprendizaje de contenidos matemáticos. Estos argumentos, son corroborados en los resultados obtenidos en el pos test del grupo experimental frente a los del grupo de control.

\section{Conclusiones}

El uso de la plataforma Khan Academy permitió fortalecer el Cálculo I en los tópicos de derivada de los estudiantes que conformaron el grupo experimental de las carreras profesionales de: Electrónica, Logística, Mecatrónica, Civil, Sistemas e Industriales, quienes obtuvieron logros académicos considerables. Además, demostraron confianza, autonomía y motivación durante el aprendizaje, consolidando sus competencias de cálculo y habilidades lógica. Desde del uso tecnológico, la manipulación de la plataforma en línea permitió rastrear las interacciones del estudiante al entrar en contacto con las herramientas e interactuar con: videos, ejercicios resueltos, desafíos de habilidades y procesos de retroalimentación que fue evidenciado en los reportes de la plataforma.

\section{Referencias}

Álvarez, C. (2018). La perspectiva generacional en los estudios de juventud: enfoques, diálogos y desafíos. Ultima década, 26(50), 40-60. https://dx.doi.org/10.4067/S071822362018000300040

Barahona, P. (2014). Factores determinantes del rendimiento académico de los estudiantes de la Universidad de Atacama. Estudios pedagógicos (Valdivia), 40(1), 2539. https://dx.doi.org/10.4067/S0718-07052014000100002

Caseres, E. A., Pereira, Z. y Pereira, L. C. (2019). Efecto del foro virtual sobre el aprendizaje de Cálculo Diferencial. Revista Electrónica de Investigación Educativa, 21(1), 1. https://doi.org/10.24320/redie.2019.21.e30.2051

Chávez, L. E. (2018). Estrategias de aprendizaje y rendimiento académico en la asignatura $\begin{array}{llll}\text { Análisis } & \text { Matemático } & \text { II. Educación, 27(53), }\end{array}$ https://doi.org/10.18800/educacion.201802.002

Delgado, I., Espinoza, J. y Fonseca, J. (2017). Ansiedad matemática en estudiantes universitarios de Costa Rica y su relación con el rendimientos académico y variables sociodemográficas. Propósitos $\quad y \quad$ Representaciones, $\quad 5(1), \quad$ 275-324. doi:http://dx.doi.org/10.20511/pyr2017.v5n1.148

Diaz, D. y Rueda, K. (2020). Use of technological tool for the consolidation of mathematical preknowledge in higher education. IOP Conference Series: Materials Science and Engineering, 844(1), 2020. https://doi.org/10.1088/1757-899X/844/1/012021

Domínguez, A., Barniol, P. y Zavala, G. (2019). Evaluación del Entendimiento Gráfico de Derivada e Integral Definida mediante un Examen en Castellano de Opción Múltiple. Formación universitaria, 12(6), 41-56. https://dx.doi.org/10.4067/S071850062019000600041

Fonseca, J. L. y Alfaro, C. R. (2018). El cálculo diferencial e integral en una variable en la formación inicial de docentes de matemática en Costa Rica. Revista Educación, 42(2), 289305. https://dx.doi.org/10.15517/revedu.v42i2.25844

Grisales, A. (2018). Uso de recursos TIC en la enseñanza de las matemáticas: retos y perspectivas. Entramado, 14(2), 198-214. https://doi.org/10.18041/19003803/entramado.2.4751 
Freiberg, A., Ledesma, R. y Fernández, M. (2017). Estilos y estrategias de aprendizaje en estudiantes universitarios de Buenos Aires. Revista de Psicología (PUCP), 35(2), 535-573. $\begin{array}{llllll}\text { Recuperado en } 17 \text { de } & \text { enero de }\end{array}$ http://www.scielo.org.pe/scielo.php?script=sci_arttext\&pid=S025492472017000200005\&lng=es\&tlng=es.

Jeong, S., Song, J. y Park, W. (2019). Analysis of student questions in MOOC physics lectures: The case of the Khan Academy. New Physics: Sae Mulli, 69(5), 484-496. https://doi.org/10.3938/NPSM.69.484

Lois, A. E., y Milevicich, L. M. (2008). La enseñanza y aprendizaje del Cálculo Integral desde la perspectiva del nuevo paradigma de la sociedad del conocimiento. Revista Iberoamericana De Educación, 47(5), 1-15. https://doi.org/10.35362/rie4752272

Lou, A. J., y Jaeggi, S. M. (2020). Reducing the prior-knowledge achievement gap by using technology-assisted guided learning in an undergraduate chemistry course. Journal of Research in Science Teaching, 57(3), 368-392. https://doi.org/10.1002/tea.21596

Quicios, M. del P., Ortega, I., y Trillo, M. P. (2016). Aprendizaje ubicuo de los nuevos aprendices y brecha digital formativa. Píxel-Bit. Revista De Medios Y Educación, (46), 155-166. https://doi.org/10.12795/pixelbit.2015.i46.10

Mondal, S., Gugnani, A., Sindhgatta, R. \& Reddy Kasireddy, V. K. (2019). Khan Academy: A social networking and community question answering perspective. IEEE International Conference on Data Mining Workshops, ICDMW, 2018-Novem, 355-359. https://doi.org/10.1109/ICDMW.2018.00058

Morales, P., Loarte, P., Alberto, C., Macuri, P., Gabriela, M. \& Macuri, P. (2018). Method Four Steps and the Learning of the Derivative By. Comuni@cción, 9(1), 48-55. http://www.scielo.org.pe/scielo.php?script=sci_arttext\&pid=S2219$71682018000100005 \& \operatorname{lng}=$ es\&tlng=es.

Muhammad, S., Hussain, M., Ge, S., Ding, H., Zhu, W., \& Zhang, W. (2020). Massive Learning Behaviours Influence Educational Sustainability: A Machine Learning Approach. Journal of Physics: Conference Series, 1487(1), 2020. https://doi.org/10.1088/17426596/1487/1/012032

Parra, Enrique, Gordillo, Wilson, \& Pinzón, Wilson J.. (2019). Modelos de Crecimiento Poblacional: Enseñanza-Aprendizaje desde las Ecuaciones Recursivas. Formación universitaria, 12(1), 25-34. https://dx.doi.org/10.4067/S0718-50062019000100025

Portilla, G. E. (2018). Modelo matemático para minimizar el número de láminas estándar y residuos metálicos durante el proceso de corte en el sector metalmecánico. Revista Logos, Ciencia \& Tecnología, 10(1). https://doi.org/10.22335/rlct.v10i1.440

Rizo, C., \& Campistrous, L. (1999). Estrategias de resolución de problemas en la escuela. Revista Latinoamericana de Investigación En Matemática Educativa RELIME, 2(3), 31-46.

Rodríguez, J., Light, D., \& Pierson, E. (2014). Khan Academy en Aulas Chilenas: Innovar en la Enseñanza e Incrementar la Participación de los Estudiantes en Matemática. In Congreso Iberoamericano de Ciencia, Tecnología, Innovación y Educación.

Rossi, R. R., Forestello, R. P., \& Recabarren, P. G. (2020). Rediseño de un programa por competencias . el caso de Electrónica Digital I • 15(29), 52-59.

Rueda, K., \& Guzmán, A. (2018). Khan Academy, una estratégia innovadora para mejorar la calidad en la educación superior a través del rendimiento académico de los estudiantes. Revista de Pedagogía, 39, 215-240. https://doi.org/10.21556/.

Siemens, G. (2005). Connectivism : a new learning theory? Journal of Instructional Technology and Distance Learning, 2(1), 1-5.

Valencia Álvarez, Adriana Berenice, \& Valenzuela González, Jaime Ricardo. (2017). ¿A qué tipo 
de problemas matemáticos están expuestos los estudiantes de Cálculo? Un análisis de libros de texto. Educación matemática, 29(3), 51-78. https://doi.org/10.24844/em2903.02

Vidergor, H. E., \& Ben-Amram, P. (2020). Khan academy effectiveness: The case of math secondary students' perceptions. Computers and Education, 157, 103985. https://doi.org/10.1016/j.compedu.2020.103985

Vrancken, S. y Engler, A. (2014). Una Introducción a la Derivada desde la Variación y el Cambio: Resultados de una investigación con estudiantes de primer año de la universidad. Bolema Mathematics Education Bulletin, 28(48), 449-468. https://doi.org/10.1590/1980$4415 \mathrm{v} 28 \mathrm{n} 48 \mathrm{a} 22$

Yassine, S., Kadry, S. y Sicilia, M. A. (2020b). Perfiles estadísticos de las interacciones de los usuarios con videos en grandes repositorios: minería del repositorio de Khan Academy. Transacciones de KSII en Internet y sistemas de información , 14, 5, (2020), 2101-2121. DOI: 10.3837 / tiis.2020.05.013. 